\title{
Using Sentiment Analysis to track reaction to the Global Game Jam Theme
}

\author{
Brian McDonald \\ Glasgow Caledonian University \\ Cowcaddens Road \\ Glasgow \\ +441413318044 \\ Brian.McDonald@gcu.ac.uk
}

\author{
David Moffat \\ Glasgow Caledonian University \\ Cowcaddens Road \\ Glasgow \\ +441413313937 \\ D.C.Moffat@gcu.ac.uk
}

\begin{abstract}
In this paper, we examine the Global Game Jam Theme and the reaction of the 'jammers' to the release. The Theme is one of the main drivers for creative aspect of the Game Jam, it sets the tone of the games that are developed at the Jam. This paper introduces an experiment which uses 'sentiment analysis' to gauge the positive or negative reaction to the theme over the last 7 years of the Global Game Jam. The results of this study show that the 2012 theme had the the highest sentiment. Finally, we suggest that the 'sentiment analysis' or 'context analysis' could be used to gather data sets for other studies such as development practices.
\end{abstract}

\section{CCS Concepts}

Information systems $\rightarrow$ Information retrieval $\rightarrow$ Specialized information retrieval $\rightarrow$ Environment-specific retrieval $\rightarrow$ Web and social media search

\section{Keywords}

Global Game Jam; Game Jam; Theme; Sentiment Analysis; Social Media; Twitter; Hash Tag; Tweets; Context Analysis

\section{INTRODUCTION}

The Global Game Jam is the largest on site game jam in the World. In 2016 there were 632 Jam sites located in 93 counties with 36,172 attendees [11].

The Global Game Jam has a common theme which is shared by every location taking part. This theme is one of the main drivers for the creative process in the Global Game Jam (GGJ). The release of this, is much anticipated by the participants of the Jam (jammers) and is the main focus of the opening presentation of each Jam site.

Permission to make digital or hard copies of all or part of this work for personal or classroom use is granted without fee provided that copies are not made or distributed for profit or commercial advantage and that copies bear this notice and the full citation on the first page. Copyrights for components of this work owned by others than ACM must be honored. Abstracting with credit is permitted. To copy otherwise, or republish, to post on servers or to redistribute to lists, requires prior specific permission and/or a fee. Request permissions from Permissions@acm.org.

GJH\&GC '16, March 13 2016, San Francisco, CA, USA (C) 2016 ACM. ISBN 978-1-4503-4083-0/16/03 ..\$15.00 DOI: http://dx.doi.org/10.1145/2897167.2897178
The theme can be a concrete statement, a phrase, an abstract concept or even a sound. It would be interesting to study the reaction to the release of this theme since the inception of the Global Game Jam and to track changes in attitude or opinion during the course of the game jam.

\section{GLOBAL GAME JAM THEME}

One of the unique elements of the Global Game Jam is the use of a central theme which is shared Globally with every site. This is released by each site at $5 \mathrm{pm}$ local time and it often triggers excitement or surprise with the jammers. Once the theme has been released the jammers start researching the theme and some sites even include pitching in the schedule for the site, for example at the Scottish Game Jam - Glasgow[19], a pitch session is scheduled 30 minutes after the release of the theme. This theme is the main design constraint of the Global Game Jam and most of the games produced over the 48-hour period will be related to the theme in some manner, either from a mechanical or thematic point of view. Given the importance of the theme it would be useful to track how people feel about it. This would allow the Global Game Jam to decide what type of theme is more popular and also track over time how well the theme is 'received'

The theme is decided by a committee of academics \& industry professionals from different nationalities and backgrounds. The theme is usually discussed months before the start of the Global Game Jam, using tools such as Basecamp or Slack. In 2012 the main three topics of discussion was ideas for the theme, the nature and role of the theme and the essence of the Global Game Jam [13]. Since 2009 the Global Game Jam theme has been one of the following [12]

Table 1. Game Jam Themes

\begin{tabular}{|l|l|l|}
\hline Year & Format & Theme \\
\hline 2009 & Statement & $\begin{array}{l}\text { As long as we have each other, we will } \\
\text { never run out of problems. }\end{array}$ \\
\hline 2010 & Word & Deception \\
\hline 2011 & Word & Extinction \\
\hline 2012 & Image & Ouroboros \\
\hline 2013 & Sound & Heartbeat \\
\hline 2014 & Statement & $\begin{array}{l}\text { We don't see things as they are, we seem } \\
\text { them as we are are. }\end{array}$ \\
\hline 2015 & Question & What do we do now? \\
\hline 2016 & Word & Ritual \\
\hline
\end{tabular}


As you can see, the theme has primarily been released in statement form or as a word. 2012 \& 2013 are the only exceptions to this, the themes from these years where delivered as an image (see figure 1) or an audio file retrospectively.

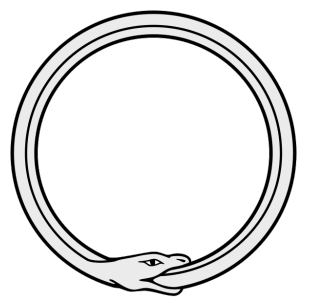

Figure 1. image of Ouroboros

The theme has the effect of constraining creativity which means that the jammers have a jumping off point to start the ideation process.

\section{SENTIMENT ANALYSIS}

Sentiment analysis (SA) is sometimes called "opinion mining", and it refers typically to the gathering of information that people write in social software platforms, and the statistical categorisation of that data according to the emotions or attitudes expressed.

The result is most simply seen as a relative proportion of sentiments expressed in the data that were either positive or negative (that is, approving or disapproving); or neither (neutral). The basic idea is a simple one, and sentiments can be judged by anybody reading the test, who knows the language and culture of the writers. The intention of the idea is usually to analyse large quantities of data however, to exploit the data that is now freely available on the internet. Data analysis gains accuracy if the quantities are large, which is why there is such interest these days for "big data." But it is expensive for human readers to do so much reading, and so researchers look for ways to write software that can do the job automatically.

The toolchain of software technologies that researchers deploy thus stretches from data gathering (using web-crawlers) to data caching and analysis, by means of natural language tools, and often with software that learns to categorise as it goes. The sources on the internet that are used can include forum comments, mailing lists and blogs; but a very important source is that form of blogging known as microblogging, where the messages are very short and to the point.

SA can be done by mining twitter feeds, and commonly is $[2,3,8$, 20], because Twitter is the most popular and famous microblogging service today.

The applications of SA are already wide and varied, even though it is still a new technology. Mining tweets, that have included geographical positioning information, has been used to identify local neighbourhoods and communities in New York City [7].

SA has been used to predict stock market price movements based on global mood swings, as expressed in twitter sentiments [4, 5, 6 14] and other blogs, including the specialist "stocktwits" microblog [15].

SA has been used in other spheres also, including politics [10], where it aims to predict the outcome of elections to office; and in market research, where it is used to detect how well a company is serving its customers. One example of this is in the airline industry, where market researchers have sought to detect how customers feel about the services that an airline provides to them [21]. In this case, an ensemble of different machine learning (ML) algorithms were applied to a twitter dataset from an airline, and found to be better together than any individual method on its own.

SA can use ML and semantic analysis to refine the accuracy of sentiments observed (as otherwise there is a lot of "noise" in the data from twitter) [9]. These techniques often use information that is sometimes present, such as emoticons, to inform the learning algorithms. They can then generalise to many more tweets (or entries without emoticons) once they have learned the emotional significance of key words in the texts. It is also possible to train the tweet-mining algorithms without providing them with any categorical information beforehand, but by using unsupervised learning techniques [16].

An important source of information about sentiments is in the meanings of the words used, and this linguistic knowledge can be included in an algorithm to enable it to understand more about the grammatical constructions and the lexicon of the language, and so achieve higher accuracy of sentiment recognition [18].

Machine learning algorithms have been applied especially for purposes of prediction, such as to the aforementioned application of the stock market, where recent historical data about prices can be very useful to estimate where the stock prices are going to go next [17].

Our method was mostly manual, and experimental, with a little support from software to use the Alchemy Sentiment API via some Javascript code. We explored two questions in our analysis: did jammers prefer some types of theme? and how did their sentiments in general change over the course of the game jam weekend?

\section{METHODS}

It is possible in Twitter to search for tweets with keywords, and with certain "hash- tags". This can be done by software, using the Twitter API, or it can be done manually, on a searching webpage on the Twitter website. As the Global Game Jam uses a different hashtag every year, we can more easily search manually for tweets relevant to that Jam.

We carried out a Twitter Search using the 'theme' as the keyword or the years theme (e.g. deception) with the following hashtags \#ggj10 \#ggj11 \#ggj12 \#ggj13 \#ggj14 \#ggj15 \#ggj16. This captured tweets from the last 7 years of the Global Game Jam, we also attempted to capture tweets from around the same date of the game jam that year, this had the impact of garnering the most immediate reactions to the theme.

As motioned previously, we could have used the Twitter API to carry out a search and then capture the data in JSON format, unfortunately the restrictions of the API meant that only Tweets form the last 7 days could be retrieved. This obviously had implications for data captured from previous game jams. This left two options, 'web scraping' search results or manual collection of tweets, because of the questionable legality of scraping it was decided to carry out manual data collection of tweet. We limited the number of tweets collected to reduce the amount of analysis required, in total 140 tweets where collected from the last 7 years of the Jam. It also should be noted that searching for the 'theme' keyword for the 2010 only provided a limited number of results, it was decided to carry out another search using keyword of 'deception'. 
The text of these tweets where then used as input to a Node.js server application which used the Alchemy API[1] to measure sentiment of the tweet. The Alchemy API uses 'deep learning' to carry out natural language processing in order to extract meta-data from content. We specifically used the sentiment analysis API from Alchemy which identifies positive/negative sentiments in the body of a given text or webpage.

The application returns back a type of sentiments which is the polarity: "positive", "negative", or "neutral" and a score where positive is greater than $\mathbf{0}$, negative is less than $\mathbf{0}$ and neutral is equal to $\mathbf{0}$. The data returned from our application is then merged with the original tweets so we can also analysis the context of the tweet.

Table 2. Sentiment Analysis - Example

\begin{tabular}{|c|c|c|}
\hline Tweet & Score & Polarity \\
\hline $\begin{array}{l}\text { Our Global Game Jam entry is officially } \\
\text { up, http://is.gd/7r15t It's quite odd, but } \\
\text { an entertaining subject, the theme was } \\
\text { deception \#GGJ10 }\end{array}$ & 0.598399 & Positive \\
\hline $\begin{array}{l}\text { This year's theme is OUT! Ritual. Happy } \\
\text { jamming! @MicrosoftNY @Playcrafting } \\
\text { @GlobalGameJam \#GGJ16 } \\
\text { \#MSPlayGGJ16 }\end{array}$ & 0.696293 & Positive \\
\hline $\begin{array}{l}\text { If I'd jammed during the \#GGJ15 with } \\
\text { this theme (what to do now?), i'd have } \\
\text { gone for a game where you have to fail } \\
\text { at everything to advance. }\end{array}$ & -0.73628 & Negative \\
\hline $\begin{array}{l}\text { Clearly we need an award for "most } \\
\text { brazen attempt to just outright ignore the } \\
\text { theme." \#GGJ12 }\end{array}$ & -0.73628 & Negative \\
\hline $\begin{array}{l}\text { The start to the \#ggj13 at } \\
\text { @GeorgeMasonU. "Just give us the } \\
\text { theme already!" }\end{array}$ & 0 & Neutral \\
\hline $\begin{array}{l}\text { For potentially obvious reasons I think } \\
\text { that Peter Gabriel should be next year's } \\
\text { GGJ theme! \#ggj12 \#worldbeat }\end{array}$ & 0 & Neutral \\
\hline
\end{tabular}

Once we collected the results from the sentiment analysis, the number of positive, negative and neutral sentiments ere counted. This is then used to gauge overall sentiment for that year's theme or the polarity of theme.

\section{INITIAL RESULTS \& DISCUSSION}

After we collected the sentiment analysis data we then calculated the polarity and net polarity for each year.

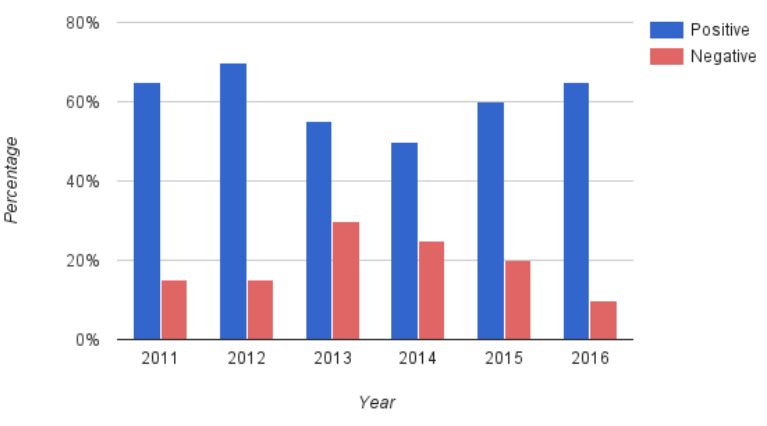

Figure 2. Theme Polarity

We found that the 2012 theme of Ouroboros had the most positive sentiment with over $70 \%$ of the tweets marked as positive by our software.

This positive sentiment for this theme could be explained by the more abstract nature of the concept that required the jammers to carry out active more research than normal. It could also mean that a theme delivered as an image could be more engaging than our mediums such as text.

It should also be noted that the 2016 theme has the least negative sentiment, it is not immediately clear why this is the case. It could be that the theme of 'ritual' is an easily identifiable word that most people could understand quite readily, which made the brainstorming process a bit easier for the jammers.

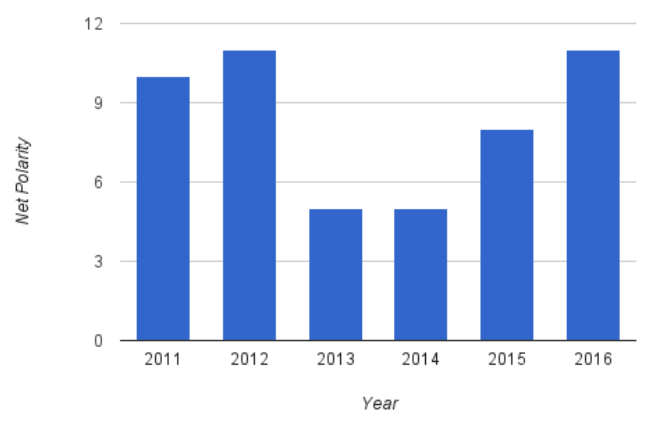

Figure 3. Theme Net Polarity

When we analyse the net polarity for each year, it was found that 2012 and 2016 both had the highest value for net polarity while 2013 and 2014 had the lowest value for net polarity. Again this seems to confirm that Jammers reacted more positively to the 2012 theme. Additional data would be require in order to carry out a proper analysis between 2012 and 2016 dates.

We omitted results from the 2010 theme as noted previously we received a limited amount of results when we used the keyword 'theme' for the search. When a search is carried out using 'deception' (the theme for that year), we received enough results to make analysis possible. It was found that $60 \%$ of tweets gathered were positive and $30 \%$ were negative for that year. 


\section{CONCLUSION}

Sentiment analysis is an excellent tool to gain insight into how a person feels about a topic or a concept. As the Global Game Jam is one of the largest game jam events in the world and there is a high volume of social media usage, then it makes sense to apply sentiment analysis to the theme or other aspects of the Game Jam. This analysis would allow Jam Organisers to see how their site is reacting and also can be used as additional data for the GGJ Theme Committee to discuss the impact of theme.

There could potentially be some issues in using sentiment analysis, as it only gives you an indication of the overall sentiment of the content. It doesn't give you a fine grained detail on each individual components in the content. This means we can only tell if the person is generally positive and in the case of the game jam this could mean they are enjoying the work that they are doing during the Jam. We would need to do further context analysis to figure out the finer detail of the jammers attitudes to the theme.

One of the attractive aspect of sentiment analysis is the lack of intrusion on the experience of the jammers \& jam organisers, other methods of gathering information from the jammers require some sort of intervention by the researcher or require active participation by the jammers.

\section{FURTHER WORK}

Since this was preliminary work it would be useful to increase the quantity of Tweets retrieved to validate the methodology explained in this paper. The sentiment analysis data could also be compared to the results from the annual Global Game Jam survey.

As mentioned in the conclusion we only have a high level measurement of the sentiment, we could bring in context analysis to measure sentiment about actual concepts in the theme. If context analysis is used we could also capture sentiment about other concepts such as development practices, feelings about the games being created and also basic feedback about the Jam sites.

It would be interesting to deploy the study to find out how jammers attitude to the theme changes over time. We could study a site such as Hawaii which is the last location to receive the theme, jammers at this site can tweet without any fears of spoiling the theme for others. This would allow us to measure in 'real time' how a site is reacting to the theme.

\section{REFERENCES}

[1] Alchemy API. http://www.alchemyapi.com/ Accessed: 201602-04

[2] Bahrainian, S.A. and Dengel, A. 2013. Sentiment analysis and summarization of twitter data. In 2013 IEEE 16th International Conference on Computational Science and Engineering.

[3] Bhuta S. and Doshi. U. 2014. A review of techniques for sentiment analysis of twitter data. In 2014 International Conference on Issues and Challenges in Intelligent Computing Techniques (ICICT).

[4] Bollen, J., Mao, H., and Zeng, X. 2011. Twitter mood predicts the stock market. Journal of Computational Science, 2(1):1-8.

[5] Corea. F. 2015. Why social media matters: The use of twitter in portfolio strategies. International Journal of Computer Applications, 128(6):25-30, October 2015. Published by Foundation of Computer Science (FCS), NY, USA.
[6] Corea, F. and Cervellati, E. M. 2015. The power of microblogging: How to use twitter for predicting the stock market. Eurasian Journal of Economics and Finance, 3(4):1-7, 2015.

[7] Deitrick, W. and Hu, W. 2013. Mutually enhancing community detection and senti- ment analysis on twitter networks. Journal of Data Analysis and Information Processing, 01(03):19-29, 2013.

[8] Garg, Y. and Chatterjee, N. 2014. Sentiment Analysis of Twitter Feeds, 2014. pages 33-52. Big Data Analytics. Springer Science + Business Media, 2014.

[9] Gautam, G. and Yadav, D. 2014. Sentiment analysis of twitter data using machine learning approaches and semantic analysis. In 2014 Seventh International Conference on Contemporary Computing (IC3), 82014.

[10] Gemilang, H. T., Erwin, A., and Eng, K. I. 2014. Indonesian president candidates 2014 sentiment analysis by using twitter data. In 2014 International Conference on ICT For Smart Society (ICISS), 92014.

[11] Global Game Jam Live Status. 2016. http://globalgamejam.org/status Accessed: 2016-02-04

[12] Global Game Jam History . http://globalgamejam.org/history Accessed: 2016-02-04

[13] Kultima, A. 2015. An Autopsy of the Global Game Jam 2012 Theme Committee Discussion : Deciding on Ouroboros. In Proceedings of the 10th International Conference on the Foundations of Digital Games, 2015.

[14] Nofer. M. 2015. Using Twitter to Predict the Stock Market: Where is the Mood Effect?, pages 63-88. The Value of Social Media for Predicting Stock Returns. Springer Science + Business Media, 2015.

[15] Oliveira, N., Cortez, P., and Areal, N. 2013. On the predictability of stock market be- havior using stocktwits sentiment and posting volume. In Progress in Artificial Intelligence, pages 355-365. Springer, 2013.

[16] Pandarachalil, R., Sendhilkumar, S. and Mahalakshmi, G. S. 2014. Twitter senti- ment analysis for large-scale data: An unsupervised approach. Cogn Comput, 7(2):254-262, 2014.

[17] Porshnev, A., Redkin, I. and Shevchenko, A. 2013. Machine learning in prediction of stock market indicators based on historical data and data from twitter senti- ment analysis. In 2013 IEEE 13th International Conference on Data Mining Workshops, 122013.

[18] Recupero, D. R., Presutti, V., Consoli, S., Gangemi A. and Nuzzolese, A. G. 2014. Sentilo: Frame-based sentiment analysis. Cogn Comput, 7(2):211-225, 2014.

[19] Scottish Game Jam - Glasgow, 2016. http://globalgamejam.org/2016/jam-sites/scottish-game-jamglasgow Accessed: 2016-02-05

[20] Venugopalan, M. and Gupta. D. 2015. Exploring sentiment analysis on twitter data. In 2015 Eighth International Conference on Contemporary Computing (IC3), page nil, 8 2015.

[21] Wan, Y. and Gao, Q. 2015. An ensemble sentiment classification system of twitter data for airline services analysis. In 2015 IEEE International Conference on Data Mining Workshop (ICDMW), 1120 